\title{
ON THE NUMBER OF COINCIDENCES OF MORPHISMS BETWEEN CLOSED RIEMANN SURFACES
}

\author{
Yolanda Fuertes and Gabino GonzÁlez-Diez ${ }^{1}$
}

\begin{abstract}
We give a bound for the number of coincidences of two morphisms between given compact Riemann surfaces (complete complex algebraic curves). Our results generalize well known facts about the number of fixed points of an automorphisin.
\end{abstract}

Let $M$ be a compact Riemann surface (complete complex algebraic curve) of genus $g \geq 2$, and $\tau: M \rightarrow M$ an automorphism different from the identity. Then it is well known (see e.g. [F-K]) that $\tau$ has at most $2 g+2$ fixed points and that this bound is attained if and only if $M$ is hyperelliptic and $\tau$ is the hyperelliptic involution.

With this in mind, we consider two distinct morphisms $f_{i}: M \rightarrow M^{\prime}$ of degrees $d_{i}(i=1,2)$ between compact Riemann surlaces of genera $g$ and $g^{\prime} \geq 2$ respectively, and look at the number of coincidences, that is, the number of points at which $f_{1}$ and $f_{2}$ agree.

The result we obtain (Theorem 2.9) is that $f_{1}$ and $f_{2}$ have at most $d_{1}+2 g^{\prime} \sqrt{d_{1} d_{2}}+d_{2}$ coincidences, and that this number (suitably counted) is attained if and only if $M^{\prime}$ is hyperelliptic and $f_{1}$ and $f_{2}$ differ by composition with the hyperelliptic involution. When these morphisms are isomorphisms, i.e. when $d_{1}=d_{2}=1$, then, of course, the coincidences are the fixed points of the automorphism $\tau=f_{1}^{-1} \circ f_{2}$; in this case our result agrees with the classical one.

The proof uses a Lefschetz trace formula for the case of two morphisms, which is a straightforward generalization of the standard one and, no doubt, is well known to topologists. However, at least in the precise form we need it here, we have not been able to locate it in the literature

\footnotetext{
${ }^{1}$ Research of the second author supported by a grant of the CICYT. M.E.C. Spain.
} 
(although see [Eich], [Lef], [K-L]); so we devote a preliminary section to establish it.

The main result is proved in Section 2; the work done there will allow us to obtain, as a byproduct, the well known theorem of de Franchis ([Fra]) on morphisms between closed Riemann surfaces.

\section{Lefschetz's trace formula}

A) In this section we first recall the basic facts in the proof of the standard Lefschetz formula for the number of fixed points of a self-mapping, and then we show how to derive, in a similar way, the Lcfschetz formula for the number of coincidences of two different mappings.

Let $M$ be a compact oricnted manifold of dimension $n$, let $\triangle \subseteq M \times M$ be the diagonal submanifold, and let $\eta_{\triangle} \in H_{D R}^{n}(M \times M)$ be its Poincare dual. For any self-mapping $f: M \rightarrow M$, the integral

$$
L(f)=\int_{M}(f \times i d)^{*} \eta_{\triangle}
$$

is called the Lefschetz number of $f$.

The classical theorem of Lefschet $z$ arises from evaluating this integral in two different ways corresponding to two different representatives of the de Rham cohomology class $\eta_{\triangle}$.

On the one hand, one has $\eta_{\triangle}=\sum_{p}(-1)^{p} \sum_{i} \pi_{1}^{*} \omega_{i}^{p} \wedge \pi_{2}^{*} \omega_{i}^{n-p}$, where $\left\{\omega_{i}^{p}\right\}$ and $\left\{\omega_{i}^{n-p}\right\}$ are basis for $H_{D R}^{p}(M)$ and $H_{D R}^{n-p}(M)$ Poincaré dual to each other, and $\pi_{i}: M \times M \rightarrow M(i=1,2)$ are the two natural projections (see e.g. $[\mathrm{B}-\mathrm{T}]$ ). This way the computation gives

$$
\begin{aligned}
L(f) & =\sum_{p=1}^{n}(-1)^{p} \sum_{i} \int_{M} f^{*} \omega_{i}^{p} \wedge \omega_{i}^{n-p} \\
& =\sum_{p=1}^{n}(-1)^{p} \text { trace } f_{\mid H^{p}(M)}^{*} .
\end{aligned}
$$

On the other hand, the Poincare dual of an oriented submanifold $Z$ of $X$ can always be represented by a form $\Phi_{Z}$, the Thom class, supported on an arbitrarily small tubular neighbourhood $T$ of $Z$ in $X$, diffeomorphic to the normal bundle $N_{Z}$ of $Z$ in $X$, with the property that the integral of $\Phi_{Z}$ along each fiber $T_{z}, z \in Z$, is 1 ( $\left.[\mathrm{B}-\mathrm{T}]\right)$. In the case of our diagonal submanifold $\triangle \subseteq M \times M$, one sees that $(f \times i d)^{*} \Phi_{\triangle}$ is supported only 
near the fixed point set of $f$, and hence, at least in the case in which $f \times i d$ is transverse to $\triangle$, we have (see $[\mathbf{B}-\mathbf{T}],[\mathbf{G}-\mathbf{H}],[\mathbf{G}-\mathbf{P}]$ )

$$
\int_{M}(f \times i d)^{*} \Phi_{\triangle}=\sum_{f(x)=x} \varepsilon(x),
$$

where $\varepsilon(x)$ is the sign of the determinant of $\left(D f_{x}-I d\right)$. We recall that $f \times i d$ being transverse to $\triangle$ is equivalent to the matrix $\left(D f_{x}-I d\right)$ being non-singular $([\mathbf{G}-\mathbf{P}])$.

More generally, let us only assume that $f$ has a finite number of fixed points (not necessarily transverse to $\Delta$ ). We recall that $L_{x}(f)$ ), the local Lefschetz number of $f$ at an isolated fixed point $x$, is defined to be the degree of the map $z \longmapsto \frac{f(z)-z}{|f(z)-z|}$ from the boundary of a small ball around $x$ to the unit sphere $S^{n-1}$.

In this situation (see $[\mathbf{G}-\mathbf{P}]$ ) one can perturb $f$ near the fixed points to obtain a map $f_{t}: M \rightarrow M$ enjoying the following properties

i) $f_{t}$ is homotopic to $f$;

ii) $f_{t}$ agrees with $f$ outside compact balls $B(x)$ around each fixed poirit $x$

iii) $\left(f_{t} \times I\right)$ is transverse to $\triangle$;

iv) $L_{x}(f)=\sum_{\left\{y \in B(x) / f_{t}(y)=y\right\}} \varepsilon(y)$.

Summing up, we obtain

$$
\begin{aligned}
L(f)=L\left(f_{t}\right) & =\sum_{f_{t}(y)=y} \varepsilon(y)=\sum_{f(x)=x} \sum_{\left\{y \in B(x) \mid f_{t}(y)=y\right\}} \varepsilon(y) \\
& =\sum_{f(x)=x} L_{x}(f) .
\end{aligned}
$$

B) The above considerations translate word for word to the case in which one has two different mappings $f_{i}: M \rightarrow M^{\prime}(i=1,2)$ between (in general, distinct) compact oriented manifolds of the same dimension.

Definition 1.1.

The Lefschetz number of two mappings $f_{i}: M \rightarrow M^{\prime}(i=1,2)$ between two compact oriented manifolds of equal dimension is defined to be

$$
L\left(f_{1}, f_{2}\right)=\int_{M}\left(f_{1} \times f_{2}\right)^{*} \eta_{\triangle}
$$

where $\eta_{\triangle}$ is the Poincaré dual of the diagonal submanifold $\triangle \subset M^{\prime} \times M^{\prime}$. 
Poincaré duality between $H_{D R}^{p}\left(M^{\prime}\right)$ and $H_{D R}^{n-p}\left(M^{\prime}\right)$ allows us to make the following

\section{Definition 1.2.}

Let $f: M \rightarrow M^{\prime}$ bc a mapping between compact oriented manifolds of the same dimension $n$. Then we shall denote by $f_{*}$ the linear map $f_{*}: H_{D R}^{p}(M) \longmapsto H_{D R}^{p}\left(M^{\prime}\right)$ determined by the property

$$
\int_{M^{\prime}} f_{*} v \wedge \omega^{\prime}=\int_{M} v \wedge f^{*} \omega^{\prime}
$$

for any $\omega^{\prime} \in H_{D R}^{n-p}\left(M^{\prime}\right)$.

Now, with the obvious notation, the analogue to (1.A) takes the following form

$$
\begin{aligned}
& \text { (1.B) } L\left(f_{1}, f_{2}\right)=\int_{M}\left(f_{1} \times f_{2}\right)^{*} \eta_{\triangle}=\sum_{p=1}^{n}(-1)^{p} \sum_{i} \int_{M} f_{1}^{*} \omega_{i}^{\prime p} \wedge f_{2}^{*} \omega_{i}^{\prime n-p} \\
& =\sum_{p}(-1)^{p} \sum_{i} \int_{M^{\prime}} f_{2 .} \circ f_{1}^{*} \omega_{i}^{\prime p} \wedge \omega_{i}^{\prime n-p}=\sum_{p}(-1)^{p} \text { trace } f_{2 .} \circ f_{1_{\mid H^{p}\left(M^{\prime}\right)}^{*}}
\end{aligned}
$$

the previous formula (1.A) being obtained by letting the second mapping be the identity. Again the form $\left(f_{1} \times f_{2}\right)^{*} \Phi_{\triangle}$ is non zero only near the coincidences of $f_{1}$ and $f_{2}$. In case $f_{1} \times f_{2}$ is transverse to the submanifold $\triangle \subset M^{\prime} \times M^{\prime}$, which again means that the matrix $\left(D f_{1, x}-D f_{2, x}\right)$ is non-singular at any such point $x$, each of these points contributes to the integral $f_{M}\left(f_{1} \times f_{2}\right)^{*} \Phi_{\Delta}$ with \pm 1 according to whether the determinant of $\left(D f_{1, x}-D f_{2, x}\right)$ is positive or negative. Thus, the analogue to (2.A) is

$$
L\left(f_{3}, f_{2}\right)=\sum_{\left\{x / f_{1}(x)=f_{2}(x)\right\}} \varepsilon(x),
$$

where $\varepsilon(x)$ is the sign of the determinant of $\left(D f_{1, x}-D f_{2, x}\right)$.

If $f_{1}, f_{2}$ satisfy the weaker condition of having a finite number of coincidences, then by perturbing $f_{1}$ in the way indicated above ([G-P]): we obtain a map $f_{t}$ enjoying the following properties

i) $f_{t}$ is homotopic to $f_{1}$;

ii) $f_{t}$ agrees with $f_{1}$ outside compact balls $B(x)$ around each of these finite number of points;

iii) $\left(f_{t} \times f_{2}\right)$ is transverse to $\Delta$;

iv) the integers $\quad \sum \quad \varepsilon(y)$ agree with the local Lefschetz $\left\{y \in B(x) / f_{t}(y)=f_{2}(y)\right\}$

numbers $L_{x}\left(f_{1}, f_{2}\right)$ (see Definition 1.3 below). 
We can now write

$$
\begin{aligned}
L\left(f_{1}, f_{2}\right) & =L\left(f_{t}, f_{2}\right)=\sum_{f_{t}(y)=f_{2}(y)} \varepsilon(y)=\sum_{f_{1}(x)=f_{2}(x)} \sum_{\left\{y \in B(x) / f_{t}(y)=f_{2}(y)\right\}} \varepsilon(y) \\
& =\sum_{f_{1}(x)=f_{2}(x)} L_{x}\left(f_{1}, f_{2}\right) .
\end{aligned}
$$

Summmarizing we have

\section{Definition 1.3.}

Let $f_{1}, f_{2}$ be as in Definition 1.1 and let $x$ be an isolated point of coincidence, then the local Lefschetz number of $f_{1}, f_{2}$ at $x, L_{x}\left(f_{1}, f_{2}\right)$, is defined to be the degree of the map $z \longmapsto \frac{f_{1}(z)-f_{2}(z)}{\left|f_{1}(z)-f_{2}(z)\right|}$ from the boundary of a small ball around $x$ to the unit sphere $S^{n-1}$.

\section{Proposition 1.4.}

Let $f_{i}: M \rightarrow M^{\prime} \quad(i=1,2)$ be two mappings between compact oriented manifolds of the same dimension $n$.

Let us assume that the set $F$ of coincidences is finite, then

$$
L\left(f_{1}, f_{2}\right)=\sum_{p=1}^{n}(-1)^{p} \text { trace } f_{1}^{*} \circ f_{2 \cdot H^{p}(M)}=\sum_{x \in F} L_{x}\left(f_{1}, f_{2}\right) .
$$

\section{Note 1.5.}

In the formula (1.B) the linear maps $f_{1}^{*}, f_{2}$. are composed in different ordcr. The change is valid bccause of the well known fact that, for any two matrices $A, B$ the traces of $A \cdot B$ and $B \cdot A$ agree, whenever the two products make sense.

Of the above sequence of traces, the first and the last ones are the easiest to work out. Let us denote by $d_{i}$ the degrec of the map $f_{i}$; then we have

\section{Lemma 1.6.}

i) $f_{1}^{*} \circ f_{2 .}: H^{0}(M) \mapsto H^{0}(M)$ is multiplication by $d_{2}$

ii) $f_{1}^{*} \circ f_{2}: H^{n}(M) \mapsto H^{n}(M)$ is multiplication by $d_{1}$.

Proof: Let us denote by $1_{M}$ and $\omega_{M}$ the standard generators of $H^{0}(M)$ and $H^{n}(M)$, respectively. Then

$$
\begin{aligned}
\int_{M^{\prime}} f_{2,} 1_{M} \wedge \omega_{M^{\prime}} & =\int_{M} 1_{M} \wedge f_{2}^{*} \omega_{M^{\prime}}=\int_{M} f_{2}^{*} \omega_{M^{\prime}}=d_{2} \\
& =\int_{M^{\prime}} d_{2} 1_{M^{\prime}} \wedge \omega_{M^{\prime}}
\end{aligned}
$$


This means that $f_{2_{n}}\left(1_{M}\right)=d_{2} 1_{M^{\prime}}$. Similarly $\int_{M^{\prime}} f_{2,} \omega_{M} \wedge 1_{M^{\prime}}=$ $\int_{M} \omega_{M} \wedge f_{2}^{*} 1_{M^{\prime}}=1=\int_{M^{\prime}} \omega_{M^{\prime}} \wedge 1_{M^{\prime}}$; which means that $f_{2} \omega_{M}=\omega_{M^{\prime}}$. From this, i) and ii) follow easily.

\section{Example 1.7.}

If either $M$ or $M^{\prime}$ is the sphere $S^{n}$, then $L\left(f_{1}, f_{2}\right)=d_{1}+(-1)^{n} d_{2}$. In particular, unless $d_{1}=(-1)^{n+1} d_{2}$, the set $F=\left\{x / f_{1}(x)=f_{2}(x)\right\}$ is nonempty.

\section{A bound for the number of coincidences}

1. In what follows we will concentrate in the case in which the manifolds $M$ and $M^{\prime}$ are compact Riemann surfaces of genera $g$ and $g^{\prime}$, and the mappings $f_{i}: M \rightarrow M^{\prime}$ are holomorphic and non constant. In this situation the Lefschetz formula of our previous section reads

$$
L\left(f_{1}, f_{2}\right)=d_{1}-\text { trace } f_{1}^{*} \circ f_{2, \mid H^{1}(M)}+d_{2} .
$$

Moreover, it is well known that the first de Rham cohomology group (with complex coefficients) splits into the direct sum of the vector spacc of holomorphic 1 -forms and its conjugate; namely

$$
H_{D R}^{1}(M, C)=\Gamma(M, \Omega) \oplus \overline{\Gamma(M, \Omega)}
$$

we have the following result

\section{Lemma 2.1.}

Let $f: M \rightarrow M^{\prime}$ be a holomorphic map between compact Riemann surfaces, and let $\tilde{f}_{*}: H_{D R}^{1}(M, \mathrm{C}) \rightarrow H_{D R}^{1}\left(M^{\prime}, \mathrm{C}\right)$ be the $\mathbf{C}$-linear map obtained by extending the $\mathbf{R}$-linear map $f_{*}$ introduced in the preceding section; then we have

i) $\tilde{f}_{*}(\Gamma(M, \Omega)) \subset \Gamma\left(M^{\prime}, \Omega\right)$;

ii) $\tilde{f}_{*}(\overline{\Gamma(M, \Omega)}) \subset \overline{\Gamma\left(M^{\prime}, \Omega\right)}$; in fact, for any holomorphic 1-form $\omega, \tilde{f}_{*} \bar{\omega}=\overline{\tilde{f}_{*} \omega}$.

Proof: Let $U^{\prime}$ be an open set of $M^{\prime}$ wcll covered by $f$. This means that $f^{-1}\left(U^{\prime}\right)$ is the disjoint union of open sets $U_{i}(i=1, \ldots, d=\operatorname{deg}(f))$ such that the restriction of $f$ to each of them is an isomorphism.

Now, given a holomorphic form $\omega$ on $M$, we assign to each such open set $U^{\prime}$ the form

$$
\omega_{\mid U^{\prime}}^{\prime}=\sum_{i=1}^{d}\left(f_{\mid U_{2}}^{-1}\right)^{*} \omega .
$$


in this way we obtain a globally well defined holomorphic form $\omega^{\prime}$ on $M^{\prime}$ (see [Spr, p. 276]).

We claim that $\omega^{\prime}=\tilde{f}_{*} \omega$. Indeed, for any 1-form $\eta$ on $M^{\prime}$ a standard partition of unity argument shows that

$$
\int_{M^{\prime}} \omega^{\prime} \wedge \eta=\int_{M} \omega \wedge f^{*} \eta
$$

The rest of the statements in the lemma follow from this fact.

Notation. At this point it is convenient to introduce a change in our notation. From now on, given a holomorphic map $f_{i}: M \rightarrow M^{\prime}$, we shall denote by $f_{i}$ the restriction of the C-linear operator $\tilde{f}_{i_{*}}$ of the lemma above to $\Gamma(M, \Omega)$. Accordingly $f_{i}^{*} \circ f_{2}$, will always denote a $\mathrm{C}$-linear endomorphism of $\Gamma(M, \Omega)$.

With this notation we have

Corollary 2.2.

$$
L\left(f_{1}, f_{2}\right)=d_{1}-\left(\text { trace } f_{1}^{*} \circ f_{2,}+\overline{\text { trace } f_{1}^{*} \circ f_{2}}\right)+d_{2} .
$$

\section{Remark 2.3.}

When $f_{i}: M \rightarrow M^{\prime} \quad(i=1,2)$ are isomorphisms, then we have $d_{1}=d_{2}=1$, and $f_{1}^{*} \circ f_{2_{n}}=\left(f_{2}^{-1} \circ f_{1}\right)^{*}$; thus, in this case, our formula is just the usual Lefschetz's formula for the automorphism $\left(f_{2}^{-1} \circ f_{1}\right)$ (sce $[\mathbf{F}-\mathbf{K}])$.

2. It is well known that the vector space $\Gamma(M, \Omega)$ carries a hermitian structure given by

$$
<v, w>=i \int v \wedge \bar{w}
$$

We have the following result

\section{Proposition 2.4.}

i) $f_{1}^{*} \circ f_{2}$, and $f_{2}^{*} \circ f_{1_{n}}$ are adjoint of each other.

ii) $f^{*} \circ f_{*}$ is self adjoint.

iii) There is an orthogonal basis $\beta=\left\{w_{1}, \ldots, w_{g}\right\}$ of $\Gamma(M, \Omega)$ with respect to which $f_{2}^{*} \circ f_{2}$. and $f_{2}^{*} \circ f_{1} \circ f_{1}^{*} \circ f_{2}$. are represented by 
the following diagonol $g \times g$ matrices of rank $g^{\prime}$

$$
\begin{gathered}
\mathcal{M}_{\beta}\left(f_{2}^{*} \circ f_{2 .}\right)=\left(\begin{array}{ccccccc}
d_{2} & 0 & \ldots & 0 & 0 & \ldots & 0 \\
0 & d_{2} & & \vdots & \vdots & & \vdots \\
\vdots & & \ddots & 0 & \vdots & & \vdots \\
0 & \ldots & 0 & d_{2} & 0 & \ldots & 0 \\
0 & \ldots & \ldots & 0 & 0 & \ldots & 0 \\
\vdots & & & \vdots & \vdots & & \vdots \\
0 & \ldots & \ldots & 0 & 0 & \ldots & 0
\end{array}\right) \\
\mathcal{M}_{\beta}\left(f_{2}^{*} \circ f_{1_{*}} \circ f_{1}^{*} \circ f_{2 .}\right)
\end{gathered}
$$

Proof: We have $<f_{1}^{*} \circ f_{2_{*}} v, w>=i \int f_{1}^{*} \circ f_{2,} v \wedge \bar{w}=$ $i \int v \wedge \overline{f_{2}^{*} \circ f_{1,} w}=\left\langle v, f_{2}^{*} \circ f_{1,} w\right\rangle$ which proves i) and ii).

In order to prove iii) we make the observation that the action of $f_{*} \circ f^{*}$ on $\Gamma\left(M^{\prime}, \Omega\right)$ is just multiplication by $d=\operatorname{deg}(f)$; this can be deduced cither from the explicit construction of $f_{*} \omega$ carried out in the proof of lemma 2.1, or from the definition given in Section 1. Indeed, for any two forms $v^{\prime}, \omega^{\prime} \in \Gamma\left(M^{\prime}, \Omega\right)$, we have $<f_{*} \circ f^{*} v^{\prime}, \omega^{\prime}>=i \int f_{*} \circ f^{*} v^{\prime} \wedge \overline{\omega^{\prime}}=$ $i \int f^{*} v^{\prime} \wedge f^{*} \bar{\omega}^{\prime}=i \int f^{*}\left(v^{\prime} \wedge \overline{\omega^{\prime}}\right)=i \cdot d \int v^{\prime} \wedge \overline{\omega^{\prime}}=\left\langle d v^{\prime}, \omega^{\prime}\right\rangle$.

This obscrvation shows that $f_{2}^{*} \circ f_{1_{*}} \circ f_{1}^{*} \circ f_{2_{*}}=d_{1} f_{2}^{*} \circ f_{2_{*}}$, and therefore it is enough to prove the statement concerning $f_{2}^{*} \circ f_{2 .}$. Now, since $f_{2}^{*} \circ f_{2}$. is self adjoint, there is an orthogonal basis $\beta$ with respect to which its matrix is diagonal. Clearly this matrix has rank at most $g^{\prime}$, but on the other hand the observation above also shows that the forms in $f_{2}^{*}\left(\Gamma\left(M^{\prime}, \Omega\right)\right)$ are all eigenvectors of $f_{2}^{*} \circ f_{2}$. with eigenvalue $d_{2}$. This completes the proof. 


\section{Corollary 2.5.}

We have the bound $L\left(f_{1}, f_{2}\right) \leq d_{1}+2 g^{\prime} \sqrt{d_{1} d_{2}}+d_{2}$. Equality holds if and only if the matrix of $f_{1}^{*} \circ f_{2}$. with respect to the basis $\beta$ above is

$$
\left(\begin{array}{ccccccc}
-\sqrt{d_{1} d_{2}} & 0 & \ldots & 0 & 0 & \ldots & 0 \\
0 & -\sqrt{d_{1} d_{2}} & & \vdots & \vdots & & \vdots \\
\vdots & & \ddots & 0 & \vdots & & \vdots \\
0 & \ldots & 0 & -\sqrt{d_{1} d_{2}} & 0 & \ldots & 0 \\
0 & \ldots & \ldots & 0 & 0 & \ldots & 0 \\
\vdots & & & \vdots & \vdots & & \vdots \\
0 & \ldots & \ldots & 0 & 0 & \ldots & 0
\end{array}\right)
$$

Proof: Let $A=\left(a_{i j}\right)$ be the matrix of $f_{1}^{*} \circ f_{2}$. with respect to the orthogonal basis above. Then, by part i) of the Proposition, ${ }^{t} \bar{A}$ will be the matrix of $f_{2}^{*} \circ f_{1_{*}}$; thus, by part iii) we have

$$
{ }^{t} \bar{A} \cdot A=\left(\begin{array}{ccccccc}
d_{1} d_{2} & 0 & \ldots & 0 & 0 & \ldots & 0 \\
0 & d_{1} d_{2} & & \vdots & \vdots & & \vdots \\
\vdots & & \ddots & 0 & \vdots & & \vdots \\
0 & \ldots & 0 & d_{1} d_{2} & 0 & \ldots & 0 \\
0 & \ldots & \ldots & 0 & 0 & \ldots & 0 \\
\vdots & & & \vdots & \vdots & & \vdots \\
0 & \ldots & \ldots & 0 & 0 & \ldots & 0
\end{array}\right)
$$

This means that

$$
\sum_{i=1}^{g}\left|a_{i k}\right|^{2}= \begin{cases}d_{1} d_{2}, & \text { if } k \leq g^{\prime} \\ 0, & \text { if } k>g^{\prime}\end{cases}
$$

From here we deduce that $\left|a_{k k}\right| \leq \sqrt{d_{1} d_{2}}$ and that equality occurs if and only if $\sum_{i=1}^{g}\left|a_{i k}\right|^{2}=\left|a_{k k}\right|^{2}=d_{1} d_{2}$. This ends the proof.

3. By Section 1, our inequality in Corollary 2.5 can be written as

$$
\begin{aligned}
L\left(f_{1}, f_{2}\right) & =\sum_{f_{1}(P)=f_{2}(P)} L_{P}\left(f_{1}, f_{2}\right) \\
& \leq d_{1}+2 g^{\prime} \sqrt{d_{1} d_{2}}+d_{2} .
\end{aligned}
$$

Now we give a convenient description of the local Lefschetz numbers $L_{P}\left(f_{1}, f_{2}\right)$. 


\section{Definition 2.6.}

Let $P \in M$ be a coincidence of $f_{1}$ and $f_{2}$; and let

$$
f_{1}(z)-f_{2}(z)=c_{k} z^{k}+c_{k+1} z^{k+1}+\cdots ; \quad c_{k} \neq 0
$$

be the Taylor expansion of $f_{1}-f_{2}$ with respect to small parametric discs $D$ of $P$ and $D^{\prime}$ of $f_{i}(P)$. We define the multiplicity of $f_{1}, f_{2}$ at $P$ to be

$$
m_{P}\left(f_{1}, f_{2}\right)=k
$$

\section{Proposition 2.7.}

Let $P \in M$ be a coincidence of $f_{1}$ and $f_{2}$; then

$$
L_{P}\left(f_{1}, f_{2}\right)=m_{P}\left(f_{1}, f_{2}\right)
$$

Proof: $L_{P}\left(f_{1}, f_{2}\right)$ is by definition the degree of the map

$$
z \in \partial D \longmapsto \frac{f_{1}(z)-f_{2}(z)}{\left|f_{1}(z)-f_{2}(z)\right|}=\frac{c_{k} z^{k}+c_{k+1} z^{k+1}+\cdots}{\left|c_{k} z^{k}+c_{k+1} z^{k+1}+\cdots\right|} .
$$

Now, if $D$ is sufficiently small, the family of maps

$$
F_{t}(z)=\frac{c_{k} z^{k}+t\left(c_{k+1} z^{k+1}+\cdots\right)}{\mid c_{k} z^{k}+t\left(c_{k+1} z^{k+1}+\cdots \mid\right.}
$$

gives a homotopy between our initial map and the map $z \rightarrow \frac{c_{k} z^{k}}{\left|c_{k} z^{k}\right|}$ which clearly has degree $k$.

Because of this proposition, in the rest of the paper we will refer to the global Lefschetz number $L\left(f_{1}, f_{2}\right)$ as the number of coincidences counted with multiplicities (or appropriately counted).

In any case, this number is always greater than or equal to the actual number of coincidences, so we have.

Corollary 2.8 .

i) $\#\left\{P \in M / f_{1}(P)=f_{2}(P)\right\} \leq d_{1}+2 g^{\prime} \sqrt{d_{1} d_{2}}+d_{2}$.

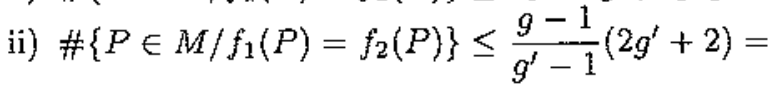

$$
2 g+2+4\left(\frac{g-1}{g^{\prime}-1}-1\right) \text {. }
$$


Proof: We only have to observe that ii) follows from i), since by means of the Riemann-Hurwitz formula $d_{i} \leq \frac{g-1}{g^{\prime}-1}$.

We observe that when $f_{1}, f_{2}$ are isomorphisms then we have $d_{1}=d_{2}=$ 1, $g=g^{\prime}$; and our bounds all equal $2 g+2=2 g^{\prime}+2$, as it should be.

4. We now address the question of whether our bound is sharp.

By Corollary .2.5., the number of coincidences $L\left(f_{1}, f_{2}\right)$ attains this bound if and only if for the first $g^{\prime}$ forms $\omega_{1}, \ldots, \omega_{g^{\prime}}$ of the orthogonal basis $\beta$, we have

$$
f_{2}^{*} \circ f_{2 .} \omega_{i}=d_{2} \omega_{i} \quad \text { and } \quad f_{1}^{*} \circ f_{2} \omega_{i}=-\sqrt{d_{1} d_{2}} \omega_{i}, \quad i=1, \ldots, g^{\prime} ;
$$

which implies that

$$
f_{1}^{*}\left(f_{2}, \omega_{i}\right)=-\sqrt{\frac{d_{1}}{d_{2}}} f_{2}^{*}\left(f_{2}, \omega_{i}\right), \quad i=I, \ldots, g^{\prime}
$$

and hence that

$$
f_{1}^{*}\left(\omega^{\prime}\right)=-\sqrt{\frac{d_{1}}{d_{2}}} f_{2}^{*}\left(\omega^{\prime}\right), \quad \text { for all } \omega^{\prime} \in \Gamma\left(M^{\prime}, \Omega\right) .
$$

It follows that the inclusions $f_{i}^{\sharp}: \mathrm{C}\left(M^{\prime}\right) \hookrightarrow \mathrm{C}(M)$ between the function fields of $M^{\prime}$ and $M$ induced by the maps $f_{2}(i=1,2)$ agree on the subfield $K \subset \mathrm{C}\left(M^{\prime}\right)$ generated by quotients of 1 -forms on $M^{\prime}$.

Let us now assume that $g^{\prime} \geq 2$; then, if $M^{\prime}$ is not hyperelliptic we have $K=\mathrm{C}\left(M^{\prime}\right)$ and, by the well known equivalence between compact Riemann surfaces and their function fields, it follows that $f_{1}=f_{2}$, which is in contradiction with $f_{1}^{*} \circ f_{2_{*}} \omega_{i}=-\sqrt{d_{1} d_{2}} \omega_{i}$.

If on the other hand $M^{\prime}$ is hyperelliptic, then $K=\mathrm{C}(x)$ is the subfield of degree 2 generated by the hyperelliptic function $x: M^{\prime} \rightarrow \mathrm{P}^{1}$ and we see that in this case either $f_{1}=f_{2}$ (which again is impossible), or $f_{2}=J \circ f_{1}$, where $J$ is the hyperelliptic involution of $M^{\prime}$ (see $[\mathrm{F}-\mathrm{K}]$ ).

Summarizing we have proved the following result

\section{Theorem 2.9.}

Let $f_{t}: M \rightarrow M^{\prime} \quad(i=1,2)$ be two morphisms between compact Riemann surfaces of genera $g$ and $g^{\prime}$ respectively, and let $L\left(f_{1}, f_{2}\right)$ denote the number of coincidences appropriately counted. We have

i) $L\left(f_{1}, f_{2}\right) \leq d_{1}+2 g^{\prime} \sqrt{d_{1} d_{2}}+d_{2}$.

ii) In case $g^{\prime} \geq 2$, this bound is attoined if and only if $M^{\prime}$ is hyperelliptic and $f_{2}=J \circ f_{1}$, where $J$ denotes the hyperelliptic involution of $M^{\prime}$. 


\section{Final remarks and examples}

\section{Example 3.1.}

Let us take as $M$ the Fermat Riemann surface of algebraic equation $x^{2 n}+y^{2 n}=1$, and as $M^{\prime}$ the hyperelliptic surface of equation $y^{2}=$ $1-x^{2 n}$; we have a map

$$
\begin{aligned}
f: M & \rightarrow M^{\prime} \\
(x, y) & \rightarrow\left(x, y^{n}\right)
\end{aligned}
$$

Let us denote by $\sigma_{i j}$ the automorphism of $M$ given by $\sigma_{i j}(x, y)=$ $\left(\xi^{i} x, \xi^{j} y\right)$, where $\xi=\exp \left(\frac{\pi \sqrt{-1}}{n}\right)$, and by $f_{i j}$ the morphism $f_{i j}=f \circ \sigma_{i j}$. In this case, we can make everything explicit. We have

$-\operatorname{deg}\left(f_{i j}\right)=n$.

$-g=(n-1)(2 n-1)$.

$-g^{\prime}=n-1$.

- The differentials $x^{r-1} y^{s-1} \frac{d x}{y^{2 n-1}}$ (with $1 \leq r, s$ and $r+s \leq 2 n-1$ ) afford a basis for $\Gamma(M, \Omega)$.

- The nonzero eigenvalues of $f^{*} \circ f_{i j}$. are $n \xi^{j n-i(k+1)}, 0 \leq k \leq n-2$; the corresponding eigenvectors being $x^{k} \frac{d x}{y^{n}}$.

$$
\begin{aligned}
-L\left(f, f_{i j}\right) & =n-n \sum_{k=0}^{n-2}(-1)^{j}\left(\xi^{-i(k+1)}+\xi^{i(k+1)}\right)+n \\
& =2 n-(-1)^{j} n\left(-1-\xi^{i n}\right) \\
& = \begin{cases}4 n ; & i \neq 0 \text { even, } j \text { even. } \\
0 ; & i \neq 0 \text { even, } j \text { odd. } \\
2 n ; & i \neq 0 \text { odd. } \\
2 n^{2} ; & i=0, j \text { odd. } \\
2 n(2-n) ; & i=0, j \text { even. }\end{cases}
\end{aligned}
$$

In any case $L\left(f, f_{i j}\right) \leq n+2(n-1) n+n=2 n^{2}$, which is the bound obtained in Theorem 2.9 ; and the bound is attained by $L\left(f, f_{0 j}\right), j$ odd. We see that $f_{03}(x, y)=\left(x,-y^{n}\right)$; thus $f_{0 j}=J \circ f$, in complete agreement with our theorem.

We also note that $L\left(f, f_{0 j}\right), j$ even, is negative as soon as $n>2$; this is because in this case $f_{0 j}$ is just $f$. 


\section{Remark 3.2.}

There is a more direct approach to estimate $\#\left\{P / f_{1}(P)=f_{2}(P)\right\}$.

Let $\varphi$ be a meromorphic function on $M^{\prime}$, then we can write

$$
\begin{aligned}
\#\left\{P / f_{1}(P)=f_{2}(P)\right\} & \leq \#\left\{P / \varphi \circ f_{1}(P)-\varphi \circ f_{2}(P)=0\right\} \\
& \leq \operatorname{deg}\left(\varphi \circ f_{1}-\varphi \circ f_{2}\right) \\
& \leq \operatorname{deg}\left(\varphi \circ f_{1}\right)+\operatorname{deg}\left(\varphi \circ f_{2}\right) \\
& =\operatorname{deg}(\varphi)\left(d_{1}+d_{2}\right) .
\end{aligned}
$$

This computation makes sense whenever $\varphi$ is such that $\varphi \circ f_{1}$ and $\varphi \circ f_{2}$ are distinct; in order to guarantee that this property is satisfied, we must allow $\varphi$ to have degrce $g^{\prime}+1$. (We recall that, by the Riemann-Roch theorem, for any $P^{\prime} \in M^{\prime}$ there exists a function of degree $g^{\prime}+1$ that takes the value $\infty$ only at the point $P^{\prime \prime}$.)

This gives us the bound $\left(g^{\prime}+1\right)\left(d_{1}+d_{2}\right)$, which is satisfactory for the automorphism case $\left(d_{1}=d_{2}=1\right)$ where we obtain the correct number $2 g+2$. However, in the general case this bound exceeds ours by $g^{\prime}\left(\sqrt{d_{1}}-\sqrt{d_{2}}\right)^{2}$.

\section{Remark 3.3.}

We note that the morphisms between the surfaces $M$ and $M^{\prime}$ cannot be replaced simply by continuous (surjective) maps ${ }^{1}$. Already when $M=$ $M^{\prime}$, one has a family of homeomorphisms $f_{n}: M \rightarrow M$ whose action on the first homology group is represented, with respect to a canonical basis, by the family of $2 g \times 2 g$ matrices

$$
A_{n}=\left(\begin{array}{cccccccc}
n & 0 & \ldots & 0 & 1 & 0 & \ldots & 0 \\
0 & n & & \vdots & 0 & 1 & & \vdots \\
\vdots & & \ddots & 0 & \vdots & & \ddots & 0 \\
0 & \ldots & 0 & n & 0 & \ldots & 0 & 1 \\
-1 & 0 & \ldots & 0 & 0 & \ldots & \ldots & 0 \\
0 & -1 & & \vdots & \vdots & \ddots & & \vdots \\
\vdots & & \ddots & 0 & \vdots & & \ddots & \vdots \\
0 & \ldots & 0 & -1 & 0 & \ldots & \ldots & 0
\end{array}\right)
$$

this is because $A_{n}$ is symplectic.

The corresponding family of Lefschetz numbers is $L\left(f_{n}\right)=2+2 g n$, which is not bounded with $g$.

Remark 3.4. (de Franchis theorem).

The work done in Section 2 also allows us to obtain the following result of de Franchis.

${ }^{1}$ We are grateful to $\mathrm{C}$. Earle for bringing this question to our attention. 


\section{Theorem.}

Let $M, M^{\prime}$ have genus $\geq 2$; then

i) The number of possible maps $f_{i}: M \rightarrow M^{\prime}$ is finite.

ii) The number of possible targets $M^{\prime}$ for fixed $M$ is finite.

We describe the proof briefly; it naturally falls into two parts:

1) First, one proves that the linear endomorphism $f_{i}^{*} \circ f_{i}$. (resp. $f_{i}^{*} \circ f_{1}, f_{1}$ kept fixed) of $\Gamma(M, \Omega)$ determines $f_{i}$ up to postcomposition with an automorphism of $M^{\prime}$ (resp. determines $f_{i}$ completely).

2) Then, one shows that there can only be finitely many such linear endomorphisms.

The proof of statement 1) is contained in the discussion of Section 2.4 that precedes Theorem 2.9. Indeed, if $f_{i}^{*} \circ f_{i .}=f_{j}^{*} \circ f_{j}$. (resp. $f_{i}^{*} \circ f_{1 *}=f_{j}^{*} \circ f_{1}$.) then the induced inclusions between function fields $f_{i}^{*}, f_{j}^{*}: \mathrm{C}\left(M^{\prime}\right) \hookrightarrow \mathrm{C}(M)$ would have the same image (resp. would coincide). Therefore, from the well known equivalence between Riemann surfaces and their function fields, we deduce that $f_{i}$ and $f_{j}$ differ by postcomposition with an automorphism of $M^{\prime}$ (resp. $f_{i}$ and $f_{j}$ agrec). Again, the case in which $M^{\prime}$ is hypcrelliptic will have to be treated separately.

In order to prove 2), we work with the cohomology group with integer coefficients $H^{1}(M, Z)$; this way we represent $f_{i}^{*} \circ f_{i_{*}}=T_{i}$ (resp. $f_{i}^{*} \circ f_{1 .}=$ $T_{i 1}$ ) by a matrix with integer entries. Then, we use Proposition 2.4.iii) to obtain that its Euclidean norm $\left\|T_{i}\right\|^{2}:=$ trace $\left(T_{i}^{*} \cdot T_{i}\right)$ is $2 d_{i}^{2} g^{\prime}$ (resp. $\left.2 d_{1} d_{i} g^{\prime}\right)$, where $T^{*}$ stands for the adjoint of the operator $T$. From this, we deduce that there is a finite number of operators $T_{i}$ (resp. $T_{i 1}$ ).

In conclusion, the finiteness of the operators $T_{i}$ (resp. $T_{i 1}$ ) proves part ii) (resp. part i)) of de Franchis theorem.

It should be said that this proof is very similar to that of $\mathrm{H}$. Martens ([Ma]) (see also [Ta], [H-S]). The only difference is that in our proof jacobians do not appear; instead we let function fields play the main role.

\section{Added on Proof.}

We have recently learnt (W. Fulton, "Intersection theory", SpringerVerlag, 1984, p. 312) that the bound given in our Theorem 2.9.i) can also be obtained by means of the intersection theory of algebraic surfaccs. Not so (as far as we can sec), the identification of the case in which this bound is attained (Theorem 2.9.ii). 


\section{References}

[B -T] R. BotT AND L. W. Tu., "Differential Forms in Algebraic Topology," G.T.M. 82, Springer, 1982.

[Eich] M. Eichler. "Lectures on Modular correspondences," Notes by S. S. Rangachari, Tata Institute of Fundamental Research, Bombay, $1955-56$.

[F-K] H. M. Farkas ANd I. KRA, "Riemann Surfaces," Springer, 1980.

[Fra] M. DE Francirs, Un teorema sulle irvolutioni irrazionali, Rend. Circ. Mat. Palermo 36 (1913), p. 368.

[G-H] P. GRIfFiths and J. HaRRIs, "Principles of algebraic geometry," Wiley, N.Y., 1978.

[G-P] V. Glillemin and A. Pollak, "Differential Topology," Prentice-Hall, 1974.

[H-S] A. Howard AND A. SOMmese, On the theorem of de Franchis, Ann. Scuola Norm. Pisa 10 (1983), 403-442.

[K-L] M. Karoubi and C. Leruste, Algebraic Topology via Differential Geometry, London Math. Soc. 99 (1989), C.U.P.

[Lef] S. Lefschetz, "Algebraic Topology," A.M.S., Providence, 1942.

[Ma] H. H. MARTENS, Observations on morphisms of closed Riemann surfaces, II. Bull. London. Math. Soc. 20 (1988), 253-254.

[Spr] G. SPRINGER, "Introduction to Riemann surfaces," Addison-Wesley, 1957.

[Ta] G. TAMME, Teilkörper höheren Geschlechtes eines algebraischen Funktionkörpers, Arch. der Math. 23 (1972), 257-259.

Departamento de Matemáticas

Universidad Autónoma de Madrid

28049 Madrid

SPAIN

Rebut el 2 de Febrer de 1993 